\title{
Adherence with the WHO's hand hygiene recommendations in an Algerian university hospital
}

\author{
Chelghoum Mustapha, Lariche Nadjet, Belbah Ismahene \\ Faculty of Medicine of Sidi Bel Abbes, University of Djillali Liabes, Algeria
}

DOI: 10.3396/ijic.v16i3.016.20

\begin{abstract}
Adherence to hand hygiene recommendations by health care workers (HCWs) participate to minimize healthcare-associated infections. There are few studies, to our present state of knowledge, which were interested in the rate of adherence in Algerian hospitals and no one on the associated factors with the non-adherence by HCWs. The objective was to determine the rate of adherence with WHO's hand hygiene recommendations (HHR) and to identify factors associated with non-adherence, in a regional university hospital. The method used was direct observation, based on the recording of hygienic actions in opportunities for HCWs according to the WHO's five indications. Observations were made from November 10, 2019 until January 5, 2020. To determine the factors associated with non-adherence, a questionnaire was administered to HCWs. The relationship between the different factors and the achievement of a hygiene action was evaluated by Pearson's Chi-square test. Five hundred three opportunities for hand hygiene were observed among $206 \mathrm{HCWs}$, during 19 observation sessions. Simple handwashing was noted in 19\% of hand hygiene actions. The overall adherence was $21 \%$. There was a wide variation in the adherence rates between the different departments and the different types of HCWs. There was a statistically significant association ( $p$ $<0.05$ ) between the availability of hygiene products, skin intolerance to hygiene products and non-adherence. The relationship between workload and adherence with HHRs was not statistically significant $(p=0.279)$. The adherence to hand hygiene recommendations remains low. Using associated factors in developing a program to improve the adherence rate will result in greater efficiency with less time and resources.
\end{abstract}

Keywords: Hand hygiene, compliance, infection control, Algeria.

Corresponding author:

Chelghoum Mustapha

Faculté de médecine, Université Djillali Liabes de Sidi Bel Abbes, Boulevard colonel Othmane,

22000 Sidi Bel Abbes, Algeria.

Email: muschelghoum@gmail.com; mustapha.chelghoum@univ-sba.dz 


\section{Introduction}

Healthcare-associated infections (HAls) occur worldwide and affect hundreds of millions of patients in both developed and developing countries. In developing countries, the risk is 2 to 20 times higher and the percentage of patients affected is sometimes more than $25 \%$. Beyond the physical and moral consequences suffered by patients and their families, HAls represent a high financial burden for health systems. ${ }^{1}$ The transmission of microorganisms through the hands of health care workers ( $\mathrm{HCWs}$ ) is recognized as the main route of spread of germs. ${ }^{2}$ Hand hygiene is the simplest and most effective measure for the prevention and control of HAls. It can be practiced by rubbing with an alcohol product or by washing with soap and water. ${ }^{3}$ Improving hand hygiene to minimize HAls is a priority of the World Health Organization (WHO). ${ }^{4}$ However, adherence of HCWs to recommended hand hygiene practices remains low in various countries around the world where the rate of adherence was less than $50 \% .{ }^{2,5,6}$ Programs to improve adherence are based on survey data that evaluate hygiene practices and identify factors associated with non-adherence with recommendations. Only one study was previously conducted in Algerian hospitals; at Bâb-El-Oued University Hospital (Algiers), it revealed a rate of 6.5\% of compliance with hand hygiene guidelines. ${ }^{7}$ There is no study, to our present state of knowledge, in other hospitals and on the associated factors with the non-adherence of hand hygiene recommendations by HCWs. The present study was. conducted by HASSANI AEK University Hospital of Sidi Bel Abbes, a regional establishment that cares for a significant number of patients. The objective was to determine the rate of adherence with $\mathrm{WHO}$ hand hygiene recommendations (HHRs) and identify factors associated with non-adherence.

\section{Material and Methods}

The study was carried out at the AEK HASSANI University Hospital, a 450-bed regional general hospital in western Algeria, in eight departments: paediatrics, medical and surgical emergencies, endocrinology, general surgery, intensive care, infant surgery, trauma, and dental department. The targeted HCWs were physicians, nurses, and auxiliaries, medical interns and other professional categories who were present with patients on survey days. HCWs included in the study were those who agreed to complete the questionnaire and be observed during care activities. Participation was voluntary and informed consent was obtained. The method used was direct observation, based on the recording of hygienic actions in opportunities for health care personnel using the five indications described by the WHO hand hygiene practical guide (before touching a patient, before an aseptic procedure, after a risk of exposure to a biological fluid, after touching a patient, and after touching a patient's environment). Observations were made from November 10, 2019 until January 5, 2020. Specially trained hospital pharmacy interns conducted the observation. Data collection was carried out on an observation form based on the WHO practical guide. Direct observation was conducted in sessions of varying duration $(60$ to 120 minutes) over the study period. The sessions were scheduled in the services during the morning period or during the most frequent care periods. In the study, a minimum of 200 opportunities was set for all five indications, in accordance with the WHO observation tools. $^{8}$

To determine the factors associated with nonadherence, a questionnaire based on the one used by WHO program was used.? The questionnaire was divided into two parts: the first part identified departments, occupational category and experience, the second part included questions related to factors associated with non-adherence with hand hygiene such as hand hygiene training, availability of hygiene products, workload, skin irritation problem. The questionnaire was administered to HCWs after a hand hygiene indication, once an individual was under observation. The HCW were considered to be observed whether they had washed hands with soap and water or used alcohol hand rub for an indication. The relationship between the different factors and the achievement of a hygiene action was evaluated by the Chi-square test. The analysis of the results was performed on SPSS version 22 (IBM, Armonk NY).

This study was approved by the Ethics Committee of the University Hospital of Sidi Bel Abbes (No. 014/ CE/DAMP/CHUSBA). 


\section{Results}

During the study period, 503 opportunities for hand hygiene were observed among $206 \mathrm{HCW}$, during 19 observation sessions. Simple hand washing was noted in 94 of the 503 opportunities (19\%) corresponding to more than $92 \%$ of hand hygiene appropriate actions; while alcohol hand rub took place at 8 of 503 occasions (2\%) corresponding to $8 \%$ of hand hygiene appropriate actions, resulting in an overall adherence of $20.28 \%$ (Table I). The mean numbers of opportunities audited per service were 63 (range 4492). There was a wide variation in the adherence rates between the different departments and the different types of HCWs.
The highest adherence was recorded in the dental department with a rate of $63.6 \%$ while the lowest adherence was recorded in the endocrinology department with a rate of $8.45 \%$. Adherence with other services ranged from $14.2 \%$ to $30 \%$ (Table I).

Paramedics: auxiliary and nurses had the highest adherence rate of $\mathrm{HH}$ recommendations with a compliance of $25 \%$ and $20.88 \%$ respectively. Other professionals (dental interns, physiotherapists, radiology technicians, and medical interns) were the category with the lowest adherence rate at $18.75 \%$. Physicians were the less compliant with a compliance rate of $15.04 \%$ (Table II).

Table I. Compliance with hand hygiene $(\mathrm{HH})$ recommendations in different departments of the Hospital University of Sidi Bel Abbes (Algeria).

\begin{tabular}{|c|c|c|c|c|c|}
\hline \multirow[t]{2}{*}{ Department } & \multirow[t]{2}{*}{ HH Opportunities } & \multicolumn{3}{|c|}{ HH appropriate Actions } & \multirow[t]{2}{*}{ HH compliance (\%) } \\
\hline & & SH wash* & HA rub * & TOTAL & \\
\hline Endocrinology & 71 & 6 & - & 6 & 8.45 \\
\hline Paediatric Emergency & 70 & 10 & - & 10 & 14.29 \\
\hline Surgical & 47 & 8 & 8 & 8 & 17.02 \\
\hline Intensive Care & 60 & 18 & - & 18 & 30.00 \\
\hline Infective surgery & 92 & 13 & - & 13 & 14.13 \\
\hline Emergency & 63 & 9 & - & 9 & 14.29 \\
\hline Trauma & 56 & 10 & - & 10 & 17.86 \\
\hline Dental & 44 & 20 & - & 28 & 63.64 \\
\hline TOTAL & 503 & 94 & 8 & 102 & 20.28 \\
\hline
\end{tabular}

* HA rub: alcohol hand rub. SH wash: wash with soap and water.

Table II. Adherence with hand hygiene recommendations according to $\mathrm{HCWs}^{*}$ categories

\begin{tabular}{lrrrr} 
HCWs & $\mathrm{N}$ & HHOs $^{*}$ & HHAs $^{*}$ & Compliance (\%) $^{*}$ \\
\hline Auxiliary & 58 & 140 & 35 & 25.00 \\
Nurses & 61 & 182 & 38 & 20.88 \\
Physicians & 40 & 133 & 20 & 15.04 \\
Others** & 47 & 48 & 9 & 18.75 \\
\hline Total & 206 & 503 & 102 & 20.28
\end{tabular}

${ }^{*}$ HCWs: Health care workers, HHOs: Hand hygiene opportunities, HHAs: hand hygiene actions.

** Others: dental interns, physiotherapists, radiology technicians, and medical interns. 
"After a risk of exposure to a biological fluid" and "after touching a patient" were the indication which generated the most actions with $31 \%$ and $25 \%$ of actions. The highest adherence rates were found in the indications "after a risk of exposure to a biological fluid" with $85.88 \%$. The lowest adherence rates were observed for the indication "before touching a patient" with $13 \%$ (Table III).

The study of associated factors (Table IV) covered 206 participants with an average age of 33 with extremes of 21 and 45 . The paediatric department was the most represented with $36 \mathrm{HCWs}$ followed by the emergency department with $31 \mathrm{HCWs}$. Nurses were the most represented with 61 people followed by auxiliaries with 58 people.

Among 206 HCWs, 141 (68\%) were not compliant with HHR. The influence of certain parameters on the compliance to HHR was studied by bivariate analysis. The majority of HCWs (70\%) reported having received specific hand hygiene training in the past,

Table III. Adherence with hand hygiene recommendations according to hand hygiene $(\mathrm{HH})$ indications

\begin{tabular}{lrrr} 
HH Indications & & HH Actions & Compliance \\
$\mathbf{N}(\%)$ & & (\%) \\
\hline Before touching a patient & $338(34)$ & $44(19)$ & 13.02 \\
Before an aseptic procedure & $161(16)$ & $31(13)$ & 19.25 \\
After a risk of exposure to a biological fluid & $85(09)$ & $73(31)$ & 85.88 \\
After touching a patient & $322(32)$ & $58(25)$ & 18.01 \\
After touching a patient's environment & $88(09)$ & $30(13)$ & 34.09 \\
\hline Total & 994 & 236 & 23.74
\end{tabular}

Table IV. Compliance with hand hygiene recommendations according to characteristics of HCWs

\begin{tabular}{|c|c|c|c|c|c|c|c|c|}
\hline \multirow{5}{*}{$\begin{array}{l}\text { Hand } \\
\text { hygiene } \\
\text { training }\end{array}$} & \multirow[b]{3}{*}{ No } & \multicolumn{6}{|c|}{ Compliance with hand hygiene recommendations } & \multirow[b]{3}{*}{0.03} \\
\hline & & \multicolumn{2}{|c|}{ Proper $\mathrm{HH}^{* *}$} & \multicolumn{2}{|c|}{ No $\mathrm{HH}$} & \multicolumn{2}{|c|}{ Total } & \\
\hline & & 12 & $(20 \%)$ & 49 & (80\%) & 61 & $(30 \%)$ & \\
\hline & Yes & 53 & $(37 \%)$ & 92 & $(63 \%)$ & 145 & $(70 \%)$ & \\
\hline & Total & 65 & $(32 \%)$ & 141 & $(68 \%)$ & 206 & $(100 \%)$ & \\
\hline \multirow{3}{*}{$\begin{array}{l}\text { Skin } \\
\text { irritation } \\
\text { problem }\end{array}$} & No & 36 & $(43 \%)$ & 47 & $(57 \%)$ & 83 & $(40 \%)$ & 0.001 \\
\hline & Yes & 29 & $(24 \%)$ & 94 & $(76 \%)$ & 123 & $(60 \%)$ & \\
\hline & Total & 65 & $(32 \%)$ & 141 & (68\%) & 206 & $(100 \%)$ & \\
\hline \multirow{4}{*}{$\begin{array}{l}\text { Availability } \\
\text { of hygiene } \\
\text { products }\end{array}$} & Rare & 50 & $(32 \%)$ & 107 & (68\%) & 157 & $(76 \%)$ & 0.003 \\
\hline & Sometimes & 10 & $(24 \%)$ & 31 & (76\%) & 8 & $(4 \%)$ & \\
\hline & Always & 5 & (63\%) & 3 & $(38 \%)$ & 41 & $(20 \%)$ & \\
\hline & Total & 65 & $(32 \%)$ & 141 & $(68 \%)$ & 206 & $(100 \%)$ & \\
\hline \multirow[t]{4}{*}{ Workload } & Minimal & 24 & (39\%) & 38 & (61\%) & 62 & $(30 \%)$ & 0.279 \\
\hline & Medium & 17 & $(27 \%)$ & 45 & (73\%) & 62 & $(30 \%)$ & \\
\hline & Important & 24 & $(29 \%)$ & 58 & (71\%) & 82 & $(40 \%)$ & \\
\hline & Total & 65 & (32\%) & 141 & (68\%) & 206 & (100\%) & \\
\hline
\end{tabular}

${ }^{*}$ Pearson's chi-square test. ${ }^{* *} \mathrm{HH}$ : Hand hygiene action 
$37 \%$ of them did not perform proper hand hygiene action. The relationship between noncompliance and training was statistically significant $(p=0.03)$.

Forty percent of HCWs had a high workload (up to 15 patients), $71 \%$ of them were non-compliant. Thirty percent of staff had a minimal workload (less than 10 patients), they had an adherence rate of $39 \%$. The relationship between workload and adherence to hygiene recommendations was not statistically significant $(p=0.279)$.

Seventy six percent of HCWs reported that hygiene products are rarely available, $68 \%$ of them were not compliant. Sixty three percent of HCWs who think that hygiene products were "always" available had performed a proper hand hygiene action. The relationship between the availability of hygiene products and the compliance to HHR was statistically significant $(p=0.003)$.

Concerning skin tolerance to hygiene products, $60 \%$ of the respondents to the questionnaire reported a skin irritation problem, $76 \%$ of them were not compliant. The correlation between the problem of skin irritation with hygiene products and nonadherence was statistically significant $(p=0.001)$.

\section{Discussion}

The results showed an overall adherence rate of $20.8 \%$. This was a higher rate than that found in a study conducted in other Algerian hospitals, which was $18 \%{ }^{10}$ In Bâb El Oued University Hospital (Algiers) the compliance was 6.5\%.7 A lower rate was also found in Morocco at $16.9 \%{ }^{10}$ and in Côte d'lvoire at $5.9 \% .^{5}$ The authors of those studies explained this low adherence rate by structural problems, in particular: the lack of hand-washbasin, hygiene product dispensers and hand drying equipment. This was noted in an earlier study conducted in the same university hospital in Sidi Bel Abbès. ${ }^{11}$ However our rate remains lower compared to other countries, Tunisia, Egypt, ${ }^{10}$ Nigeria $^{12}$ and other Middle Eastern countries ${ }^{13,14}$ which have shown an adherence rate between 32.3 and $90 \%$.

Hand rub with alcohol products was the least used technique by HCWs, compared to simple hand washing with a very low rate of $2 \%$. This may be explained by the availability of sinks and water in the different patient's rooms, more than applicators of alcohol handrub. The perception by HCW of handwashing or alcohol rub may be different. This finding was different from data found in many studies, where the use of alcohol handrub is preferred. For example, in a study conducted in a national hospital in Mali showed an overall proportion of hand rubbing of $93.3 \% .{ }^{15}$ In that study, the observation was done after a promotional program of hand hygiene recommendations.

The highest adherence with hand hygiene practices was found in intensive care department $(36 \%)$ and dental department (63.6\%). Our results are consistent with those found in Algeria at the Bâb El Oued University Hospital, where adherence was higher in intensive care units $(15.8 \%)$ than in other medical services. ${ }^{7}$ However, these rates diverge from the results of a study reported at Geneva's university hospitals, where adherence was lower in intensive care units (36\%) than in internal medicine (59\%). ${ }^{2}$

Hand hygiene adherence during the assessment period was highest among the category represented by dental interns, medical interns, nurses and auxiliaries with close rates ranging from $(29 \%$ to $33.3 \%)$. The physician category was the least compliant (20\%). This finding is consistent with that reported in a university hospital in Nigeria ${ }^{12}$ which found low adherence rates among physicians (59.7\%) compared to other paramedical and auxiliary professionals (72.9\%), and also in Nottingham UK where the adherence was $47 \%$ for doctors, $75 \%$ for nurses, $78 \%$ for paramedics and $59 \%$ for auxiliary and other staff. ${ }^{16}$ The same observation was noted in Geneva's university hospitals. ${ }^{2}$ However, the category of physicians was more compliant in Mali's hospital. ${ }^{15}$

The adherence rates for the five WHO's moments were variable. The highest rate was observed for the indications "after a risk of exposure to a biological liquid" at $86 \%$ and the lowest for "before touching a patient" at $13 \%$. Our result was similar to those reported in a recent study in Germany, where adherence rates before contact with the patient with aseptic tasks were lower (17-47\%) than after contact with the patient, his fluid body or his environment 
(31-78\%). ${ }^{17}$ The same result was cited by Randle et al in England, who observed that adherence "after exposure to a body fluid" was higher (93\%) compared to other hand hygiene indications. ${ }^{16}$

HCWs after contact with a patient or body fluid felt that the risk of infection is high, hence this hand hygiene action. The risk before contact with the patient and his environment and the notion of contamination are underestimated. The caregiver tends to protect himself or herself first. The perception of nosocomial risk by HCWs is unequal and there is an inaccuracy in the knowledge of risk factors, hand washing, and the use of gloves. ${ }^{18}$

The composition of HCWs sample was variable in different studies; some were mainly intensive care staff, ${ }^{17}$ or from surgical departments. ${ }^{7}$ In other studies, the observation was performed after a hand hygiene promotional program. ${ }^{12}$

The secondary objective of this study was to determine the factors associated with nonadherence with hand hygiene recommendations, to contribute to the development of programs for their improvement. Seventy percent of respondents reported having received hand hygiene training. This rate was higher than what was reported in the Malian study with a rate of $55.8 \%{ }^{15}$ and the Tunisian's one where a rate of $31 \%$ was found. ${ }^{19}$ However, $63 \%$ of the previously trained staff were not compliant. This pre-service training showed a statistically significant correlation ( $p=0.03$ ) with non-adherence. This finding is contradictory, as training should improve hand hygiene practices. This alleges that this training did not achieve its objective of modifying and improving their knowledge and behaviour. The requirements of the staff on the risk related to care, whether for the patient by cross-contamination (HAls) or himself (occupational accidents), are not correct. The high value of adherence after contact with the patient or his biological fluid and very low before contact indicates that staff promotes protective practices over hygiene practices. It seems necessary to first evaluate the staff's knowledge of hospital hygiene to determine the objectives of the training. Then, provide periodic training on hygiene indications and infectious risk and distinguish between the concepts of personal protection to prevent occupational accidents and hygiene to avoid patient contamination.

Regarding the availability of hygiene products, $68 \%$ of non-compliant staff reported that the availability of hygiene products was rare and that the supply was not constant. They saw this as an obstacle to good hand hygiene. The association between the availability of hygiene products and non-adherence was statistically significant $(p=0.003)$. This result is in line with that reported by the Tunisian study, where the unavailability of hygiene products was frequently mentioned by $84.8 \%$ of employees. ${ }^{19}$ The importance of easy access to hand hygiene products, whether it is hand washing, soap, alcohol solution, is obvious. It is felt that asking busy staff to move away from the patient's bed to a dispenser of antiseptic solutions does not facilitate the application of hand hygiene recommendations. The department managers must inform his staff about the availability of hand hygiene products, and ensure their easy access. A product that is not within the reach of the staff is not necessarily out of stock. During the study, the presence of products was not ensured for each staff member, mainly because of non-individual packaging. The service coordinators held the products and did not systematically deliver them to the health care staff. It is therefore advisable to obtain the alcohol solution in small packages and deliver it directly to the HCWs. Coordinators should be informed about the availability of alcohol solutions and made aware of their role in monitoring and controlling their use.

Another influential factor is skin intolerance to hygiene products. Sixty percent of respondents reported having had a skin irritation problem for the hygiene products used (bleach in particular, used mixed with water in certain departments) of which $76 \%$ were not compliant. The association between the occurrence of a skin irritation problem and non-adherence was statistically significant $(p=0.001)$. Various previous studies are in agreement with our results. These studies addressed the issue of skin irritation from hand hygiene products as part of the adherence issue. In a study carried out at the Centre Hospitalier Universitaire de Lyon in France, tests were carried out under working conditions to measure the tolerance and acceptability of hand disinfectants available on the 
market; dryness and skin irritation were noted after each test period..$^{20}$ In another multicentre study in the university hospitals of New York, Lyon, and Geneva, $85 \%$ of the audited staff reported having symptoms or signs of dermatitis on their hands because of the use of antimicrobial soaps. ${ }^{20}$ The frequent and repeated use of hand hygiene products, particularly soaps and other detergents is a major cause of chronic irritant contact dermatitis among health professionals, which limits their adherence to hand hygiene practices. Adverse skin reactions can be avoided by using alcohol-based preparations containing an emollient that helps protect the skin against dry skin. Other reasons given as difficulties encountered by HCWs for good hand hygiene practice was the lack of time due to the high workload. Sixty percent of staff rated their workload as between medium and low. Sixty seven percent of them were not compliant. Statistical analysis of the relationship between workload and non-compliance revealed a statistically non-significant association $(p=0.279)$. While some previous studies have cited workload as a barrier to compliance, these studies did not statistically measure the association between workload and non-compliance. ${ }^{21}$ Our result is consistent with a study conducted in an emergency department in Canada where patient volume was not associated with non-compliance, while the length of working hours was. ${ }^{22}$

\section{Conclusion}

Overall compliance to hand hygiene recommendations has been assessed. In the Sidi Bel Abbes University Hospital, the adherence rate was low, indicating that this establishment is not exempt from the international trend. Some previous studies and in particular at the SBA University Hospital did not use the direct observation method which is the most recommended by WHO. The present study measured the association between non-adherence and certain factors. Defective or absent training, availability and quality of hygiene products were associated with it. These factors should be the elements of interventions to improve adherence. The results of our study can be improved by extending the observation to other services and by assessing the quality and appropriateness of hand hygiene techniques; the count of actions in our study did not consider this factor. The results of our study open the research to other perspectives: evaluating the extent of the presence of HAls in the current state where hygiene standards are not respected will help determine the usefulness of intervention programs; and conducting an economic evaluation of the intervention program and assessing compliance after its implementation.

\section{Conflict of Interest}

The authors declare no conflicts of interest. All material is original and not previously or currently considered for publication elsewhere. All authors have been involved in all aspects of this study.

\section{References}

1. World Health Organization. Guide de mise en oeuvre de la stratégie multimodale de l'OMS pour la promotion de l'hygiène des mains. Genève: Organisation mondiale de la Santé; 2010.

2. Pittet D. Hand hygiene: revolution and globalization. Rev Med Suisse 2009; 5(197): 716-8, 720-721.

3. Dietl B, Calbo E. Top-ten papers in infection control (20152017). Rev Esp Quimioter 2018; 31(Suppl 1): 62-65.

4. Kampf G, Löffler H, Gastmeier P. Hand hygiene for the prevention of nosocomial infections. Dtsch Arztebl Int 2009; 106(40): 649-655. https://doi.org/10.3238/ arztebl.2009.0649

5. Coulibaly-Koffi NT, Tea-Diop Y, Kouadio-Kone F, Guinan JC. Observance des pratiques d'hygiène des mains aux services du CCTOS de pédiatrie et d'ORL du CHU de Cocody. Rev IV Odonto Stomatol 2012; 14(1): 6-14.

6. Girou E, Chai SHT, Oppein F, et al. Misuse of gloves: the foundation for poor compliance with hand hygiene and potential for microbial transmission. J Hosp Infect 2004; 57(2): 162-169. https://doi.org/10.1016/j.jhin.2004.03.010

7. Makhlouf $F$, Nourredine $D$. Etude de l'impact de l'hygiène des mains sur l'incidence des infections du site opératoire au niveau des services de chirurgie du CHU Bab El Oued (2010-2011) [PhD Thesis]. 2016.

8. Réseau CClin/Arlin - Grephh. Guide méthodologique. Observance de l'hygiène des mains et Qualité de la friction. 2016;1-33.

9. Kilpatrick C. Save Lives: Clean Your Hands. A global call for action at the point of care. Am J Infect Control 2009; 37(4): 261-262. https://doi.org/10.1016/j.ajic.2009.02.001

10. Amazian K, Abdelmoumene T, Sekkat $S$, et al. Multicentre study on hand hygiene facilities and practice in the Mediterranean area: results from the NosoMed Network. $J$ Hosp Infect 2006; 62(3): 311-318. https://doi.org/10.1016/j. jhin.2005.09.023

11. Beghdadli B, Belhadj Z, Chabane W, Ghomari O, Kandouci $A B$, Fanello $S$. 'Standard precautions' practices among nurses in a university hospital in Western Algeria. Sante Publique (Vandoeuvre--Nancy France) 2008; 20(5): 445453. https://doi.org/10.3917/spub.085.0445 
12. Uneke CJ, Ndukwe CD, Oyibo PG, Nwakpu KO, Nnabu $\mathrm{RC}$, Prasopa-Plaizier N. Promotion of hand hygiene strengthening initiative in a Nigerian teaching hospital: implication for improved patient safety in low-income health facilities. Braz J Infect Dis 2014; 18(1): 21-27. https://doi. org/10.1016/j.bjid.2013.04.006

13. Marjadi B, McLaws M-L. Hand hygiene in rural Indonesian healthcare workers: barriers beyond sinks, hand rubs and inservice training. J Hosp Infect 2010; 76(3): 256-260. https:// doi.org/10.1016/j.jhin.2010.06.021

14. Arias AV, Garcell HG, Ochoa YR, Arias KF, Miranda FR. Assessment of hand hygiene techniques using the World Health Organization's six steps. J Infect Public Health 2016; 9(3): 366-369. https://doi.org/10.1016/j.jiph.2015.11.006

15. Bengaly L, Fomba S, Keita M, Traoré AT, Fofana A, Dembélé JP. Impact de l'usage des gants médicaux sur l'observance de I'hygiène des mains au cours des soins au Centre Hospitalier et Universitaire du Point $G$ de Bamako. Rev Malienne D'Infectiologie Microbiol. 2016;Tome 7:33-38.

16. Randle J, Arthur A, Vaughan N. Twenty-four-hour observational study of hospital hand hygiene compliance. J Hosp Infect 2010; 76(3): 252-255. https://doi.org/10.1016/j. jhin.2010.06.027
17. Scheithauer S, Oberröhrmann A, Haefner $\mathrm{H}$, et al. Compliance with hand hygiene in patients with meticillin-resistant Staphylococcus aureus and extended-spectrum $\beta$-lactamaseproducing enterobacteria. J Hosp Infect 2010; 76(4): 320323. https://doi.org/10.1016/j.jhin.2010.07.012

18. Trop M. Perception du risque nosocomial parmi le personnel hospitalier de l'Hôpital Principal de Dakar (2004). Med Trop (Mars) 2008; 68(6): 593-596.

19. Saidani M, Ennigrou S, Soltani H, Ben Redjeb S. Questionnaire evaluation of hand hygiene by the healthcare staff of Charles Nicolle Hospital of Tunis. Tunis Med 2010; 88(10): 731-736.

20. Larson E, Girard R, Pessoa-Silva CL, Boyce J, Donaldson L, Pittet $D$. Skin reactions related to hand hygiene and selection of hand hygiene products. Am J Infect Control 2006; 34(10): 627-635. https://doi.org/10.1016/j.ajic.2006.05.289

21. Birgand G, Lucet J-C. Comportements des soignants vis-à-vis du risque infectieux: quelles influences et comment impulser le changement? J Anti-Infect 2016; 18(1): 19-28. https://doi. org/10.1016/j.antinf.2016.01.009

22. Muller MP, Carter E, Siddiqui N, Larson E. Hand hygiene compliance in an emergency department: the effect of crowding. Acad Emerg Med 2015; 22(10): 1218-1221. https:// doi.org/10.1111/acem.12754 